\title{
M2b macrophages protect against myocardial remodeling after ischemia/reperfusion injury by regulating kinase activation of platelet-derived growth factor receptor of cardiac fibroblast
}

\author{
Yuan Yue ${ }^{1,2 \#}$, Suiqing Huang ${ }^{1 \#}$, Huayang $\mathrm{Li}^{1 \#}$, Wei $\mathrm{Li}^{3}$, Jian Hou ${ }^{1}$, Li Luo ${ }^{1}$, Quan Liu ${ }^{1}$ Cuiping Wang ${ }^{4}$, \\ Song Yang ${ }^{4}$, Linhua $\mathrm{Lv}^{1}$, Jinghua Shao ${ }^{5}$, Zhongkai $\mathrm{Wu}^{1,2}$ \\ ${ }^{1}$ Department of Cardiac Surgery, First Affiliated Hospital, Sun Yat-sen University, Guangzhou, China; ${ }^{2}$ NHC Key Laboratory of Assisted \\ Circulation, Sun Yat-sen University, Guangzhou, China; ${ }^{3}$ Department of Medical Ultrasound, First Affiliated Hospital, Sun Yat-sen University, \\ Guangzhou, China; ${ }^{4}$ Department of Cardiothoracic Surgery ICU, First Affiliated Hospital, Sun Yat-sen University, Guangzhou, China; ${ }^{5}$ Out-Patient \\ Department, Shenxian Second People's Hospital, Shenxian, China \\ Contributions: (I) Conception and design: Y Yue; (II) Administrative support: Z Wu; (III) Provision of study materials or patients: Y Yue, Z Wu; (IV) \\ Collection and assembly of data: Y Yue, S Huang, H Li, W Li, Q Liu; (V) Data analysis and interpretation: Y Yue, S Huang, J Hou, L Luo, C Wang; \\ (VI) Manuscript writing: All authors; (VII) Final approval of manuscript: All authors. \\ \#These authors contributed equally to this work. \\ Correspondence to: Zhongkai Wu, MD, PhD. Department of Cardiac Surgery, First Affiliated Hospital, Sun Yat-sen University, 58 Zhongshan II Road, \\ Guangzhou, China. Email: wuzhk@mail.sysu.edu.cn.
}

Background: Myocardial injury is a major cause of myocardial remodeling. Macrophages are important in cardiac repair as a result of their interactions with fibroblasts. As regulatory macrophages, M2b macrophages modulate inflammatory immune responses without participating in wound healing and could have enhanced protective effects on myocardial remodeling. Therefore, we tested the hypothesis that M2b macrophages could improve cardiac function and ameliorate myocardial fibrosis after the myocardial ischemia/reperfusion injury (MI/RI).

Methods: In vivo, MI/RI models were established with Sprague-Dawley (SD) rats and either M2b macrophages (MT group) or the same volume of vehicle (CK group) was injected into the ischemic zone. Two weeks after the operation, cardiac function and diameters were determined by echocardiography examination. Level of myocardial fibrosis was measured by Sirius red staining and the expression of fibrosisrelated factors. In vitro, cardiac fibroblasts (CFs) were co-cultured with $\mathrm{M} 2 \mathrm{~b}$ macrophages or cultured with M2b macrophage supernatant. Expression of $\alpha$-smooth muscle actin ( $\alpha$-SMA) and connective tissue growth factor (CCN2/CTGF) in the CFs were measured by western blotting and immunofluorescence staining. In addition, the expression of platelet-derived growth factors (PDGFs), the expression of platelet-derived growth factor receptors (PDGFRs) and the phosphorylation of PDGFRs was detected by western blotting.

Results: A significantly higher rat survival rate, improved left ventricular (LV) systolic function, decreased diameter of the LV and alleviated myocardial fibrosis were observed in the MT group than in the CK group. In vitro, the activation of CFs was significantly reduced by the M2b macrophages treatments, relative to the blank control. In addition, the kinase activation of PDGFRs was decreased by M2b macrophage treatments both in vivo and in vitro.

Conclusions: Our study demonstrated that the administration of M2b macrophages could attenuate myocardial remodeling after MI/RI. The regulation of the activation of PDGFRs in CFs is an important part of the protective mechanism.

Keywords: M2b macrophage; myocardial remodeling; cardiac fibroblast (CF); myocardial ischemia/reperfusion injury (MI/RI) 
Submitted Mar 23, 2020. Accepted for publication Aug 27, 2020.

doi: 10.21037/atm-20-2788

View this article at: http://dx.doi.org/10.21037/atm-20-2788

\section{Introduction}

The restoration of blood flow after cross-clamping in cardiopulmonary bypass (CPB) during cardiac surgery and revascularization therapy after myocardial infarction (MI) can trigger a series of events, including tissue injury, called myocardial ischemia/reperfusion injury (MI/RI). MI and MI/RI are the most common causes of cardiac fibrosis, resulting in myocardial remodeling and heart failure. The proliferation of cardiac fibroblasts (CFs) and their conversion into synthetic myofibroblasts (MFs) are hallmarks of this process $(1,2)$. There is accumulating evidence that macrophages are the key orchestrators of fibrosis through their interactions with fibroblasts (3-6).

Macrophages are heterogeneous and are characterized by having great plasticity in response to environmental signals $(7,8)$, and it is increasingly recognized that the polarized M1/M2 classification clearly represents an oversimplification (9). Mosser and Edwards have suggested a functional classification of macrophages that distinguishes, in addition to the classically activated cells, subpopulations of regulatory and reparative macrophages. Based on previous studies of heart damage and repair, classically activated (M1) macrophages are believed to damage the heart in the early period of reperfusion by releasing reactive oxygen species (ROS), inflammatory mediators and proteases $(2,10)$. Wound healing macrophages [the M2a/c subclass (9)] could be beneficial in early injury stages but deleterious during the late phase of scar formation and ventricular remodeling (11). M2b macrophages, which were defined as regulatory macrophages (9), have potential as anti-inflammatory cells, which can reduce immune responses and cause little fibrosis. Unfortunately, experimental studies investigating the role of the $\mathrm{M} 2 \mathrm{~b}$ macrophage subpopulations in the healing of ischemia/reperfusion (I/R) injuries have not been performed.

Our previous study on M2b macrophages in cardiac injury demonstrated that the transplantation of $\mathrm{M} 2 \mathrm{~b}$ macrophages is a novel approach to ameliorate tissue injury in the setting of MI/RI (12). In the current study, we evaluated the effects of M2b macrophages on myocardial fibrosis 2 weeks after I/R injury. In addition, to eliminate the ameliorated fibrosis associated with the mitigation of the acute injury, we investigated whether M2b macrophages can modulate $\mathrm{CF}$ activation directly. Kinase activation of platelet-derived growth factor receptors (PDGFRs), which is the key driving forces in CFs activation and differentiation to generate MFs $(1,13)$, was also detected both in vivo and in vitro.

We present the following article in accordance with the ARRIVE reporting checklist (available at http://dx.doi. org/10.21037/atm-20-2788).

\section{Methods}

A detailed account of the Materials and Methods used in this study is provided in the Supplementary file (Appendix 1). Briefly, M2b macrophages were polarized from bone marrow-derived macrophages (BMDMs) of Sprague-Dawley (SD) rats by lipopolysaccharide and IgG as described previously (12). CFs were enzymatically isolated from the hearts of adult male SD rats as previously described (14). In vivo, myocardial I/R injury models were established with the same strain of rats, and either $1 \times 10^{6}$ M2b macrophages (MT group) or the same volume of normal saline (CK group) was injected into the ischemic zone. Rats in the sham operation (SO) group underwent the operation without ligation of the coronary artery. Rats that experienced severe arrhythmia, cardiac arrest, or respiratory failure during the procedure were excluded. There were four, four and two rats that showed these effects in the CK, MT and SO groups, respectively.

Two weeks after the operation, cardiac function and diameters were detected by echocardiography examination. The level of myocardial fibrosis was measured by Sirius red staining and real-time quantitative polymerase chain reaction (RT-qPCR). In vitro, CFs were cocultured with the M2b macrophages or were cultured with M2b macrophage supernatant. The expression of $\alpha$-smooth muscle actin ( $\alpha$-SMA) and connective tissue growth factor (CCN2/CTGF) in CFs were measured by western blotting and immunofluorescence staining. In addition, the expression of platelet-derived growth factors (PDGFs), expression of PDGFRs and the phosphorylation of PDGFRs were detected by western blot analysis. Experiments were performed under a project license 
(No. gdpulacSPF2017026) granted by the Animal Ethical Committee of Guangdong Pharmaceutical University, in compliance with the relevant guidelines for the care and use of laboratory animals.

\section{Statistical analysis}

Data are presented as the mean \pm SD. Statistical analysis was performed using GraphPad Prism software and SPSS. The differences in the results between two groups were analyzed with Student's $t$-test. Comparisons among groups were assessed with one-way ANOVA followed by Bonferroni's post hoc test. For all tests, $\mathrm{P}<0.05$ was considered statistically significant.

\section{Results}

\section{Transplantation of M2b macrophages increased survival and improved cardiac function after I/R injury}

The results of flow cytometry showed that $89.30 \%$ of the cells were tumor necrosis factor superfamily member 14 (LIGHT/TNFSF 14 ) $^{+} \mathrm{CD} 5^{+}$(markers of $\mathrm{M} 2 \mathrm{~b}$ macrophages) after stimulation, indicating that most BMDMs were polarized into M2b macrophages (Figure 1A). Rats in the MT group (M2b macrophage transplantation) and SO group (sham operation) had a significantly higher survival rate than those in the CK group (control check) after MI/R (Figure 1B). Rats subjected to I/R injury (CK group) showed significantly worse cardiac function, which was assessed by left ventricular ejection fraction (LVEF), compared to that in the SO group. M2b macrophage transplantation significantly improved left ventricular (LV) function (Figure 1C,D, Table 1) compared to that in the CK group. In addition, compared to the CK group, myocardial remodeling was decreased by cell transplantation, which was demonstrated by LV enlargement and hypertrophy (Table 1).

\section{Transplantation of M2b macrophages alleviated myocardial fibrosis after I/R injury}

Cardiac fibrosis was evaluated with Sirius red staining and RT-qPCR. Heart sections were stained with Sirius red and observed under a light microscope. The proportion of fibrosis relative to the total heart area was referred to as the fibrosis area. Sirius red staining showed the most collagen deposition in the ischemic area, and there was some staining in the nonischemic area (Figure 2A). As shown in
Figure 2B, I/R injury significantly increased collagen deposition, compared to that in the SO group, which was attenuated by $\mathrm{M} 2 \mathrm{~b}$ macrophage transplantation. In addition, the mRNA levels of the fibrosis-related genes [collagen I, collagen III, transforming growth factor- $\beta$ (TGF- $\beta$ ), CCN2, and hepatocyte growth factor (HGF)] are shown in Figure $2 C$. The I/R injury significantly increased the expression of these genes when compared to vehicle treatment, and M2b macrophage transplantation attenuated the increases of collagen I, collagen III and TGF- $\beta$ compared to those in the CK group.

\section{$M 2 b$ macrophages influenced the expression of PDGFs and the kinase activation of PDGFRs in beart tissue}

The protein expression of PDGFs, PDGFR $\alpha$, PDGFR $\beta$ and their phosphorylated forms (p-PDGFR $\alpha$ at Tyr720; p-PDGFR $\beta$ at Tyr740) in myocardial tissue were tested by western blotting. As shown in Figure $3 A, B$, I/R injury significantly increased the expression of PDGFc and PDGFd, compared to that in the SO group, which was attenuated by $\mathrm{M} 2 \mathrm{~b}$ macrophage transplantation. The same trend of changes were observed in the expression of PDGFa and PDGFb, but without statistical significance. The expression levels of PDGFR $\alpha$ and PDGFR $\beta$ were decreased in the CK and MT groups compared to the SO group, and no difference was observed between the CK and MT groups, as shown in Figure 3A,C. The phosphorylation level (Phos-PDGFR/PDGFR) of PDGFR $\beta$ was higher in the CK group than in the SO group. Lower levels of PDGFR $\alpha$ and PDGFR $\beta$ phosphorylation (Phos-PDGFR/PDGFR) were observed in heart tissues following M2b macrophage transplantation compared to those in the CK group, which indicated that the activation of PDGFRs was decreased.

\section{$M 2 b$ macrophages inhibited the kinase activation of PDGFRs of CFs in vitro}

CF activation was assessed by the expression of $\alpha$-SMA and CCN2. Immunofluorescence staining showed that the expression of $\alpha$-SMA, a marker of differentiation into MFs, was decreased both by co-culturing with M2b macrophages and culture with the supernatant, compared to that of the blank control (NT) (Figure 4A). As detected by western blotting, the expression of $\alpha$-SMA and CCN2 in CFs were decreased both by co-culturing with M2b macrophages and culturing with the supernatant compared to that of the blank control (Figure 4B). Then, the expression 
A

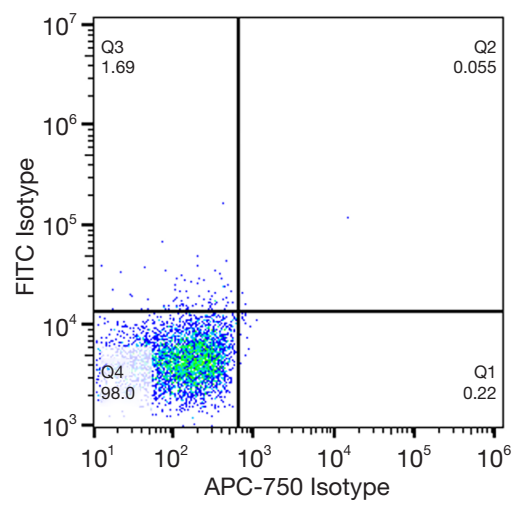

\section{B}

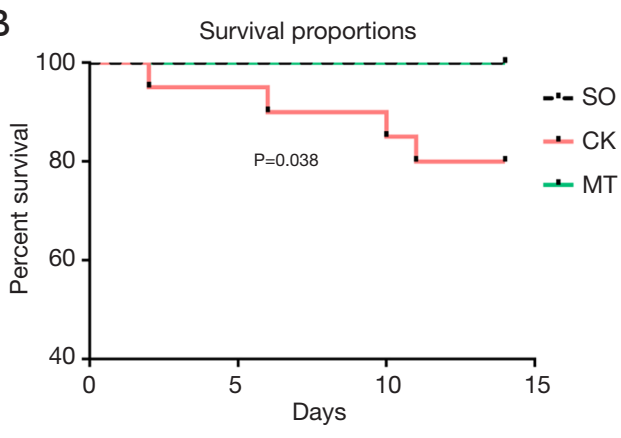

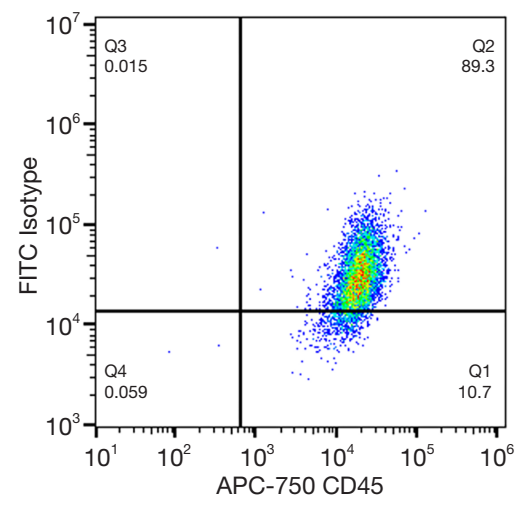

D

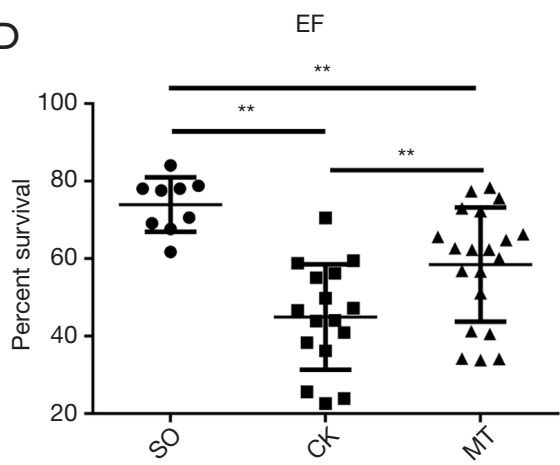

C

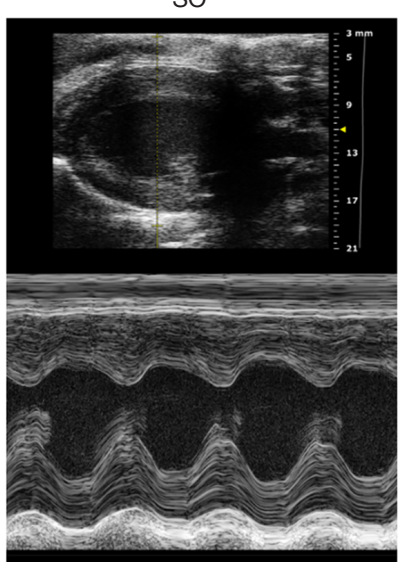

CK

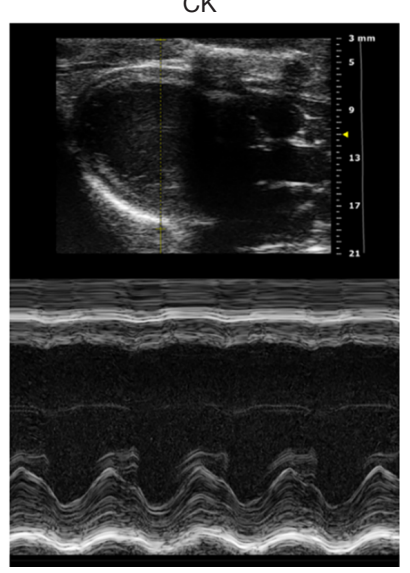

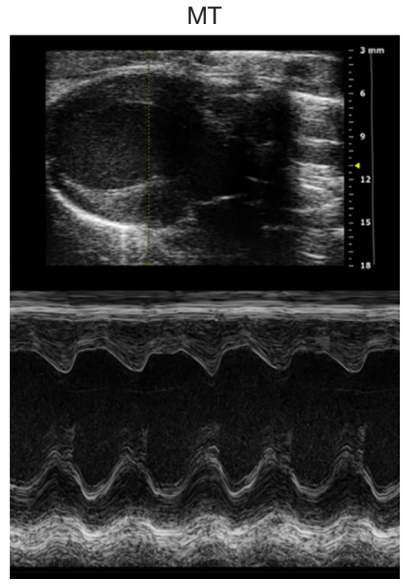

Figure 1 Transplantation of M2b macrophages increased survival and improved cardiac function after I/R injury. In rats that underwent the myocardial I/R procedure, either $1 \times 10^{6} \mathrm{M} 2 \mathrm{~b}$ macrophages (MT group) or the same volume of normal saline (CK group) were injected into the ischemic zone. Rats in the sham operation (SO) group underwent the operation without ligation of the coronary artery. (A) M2b macrophages were stained to assess LIGHT and CD45 expression and were analyzed by flow cytometry; (B) Kaplan-Meier survival curves after MI/R in the different groups (rat numbers at 0, 5, 10, and 14 days after MI/R: SO, 10, 10, 10 and 10; CK, 20, 19, 17 and 16; and MT, 20, 20, 20 and 20, respectively); (C) M-mode echocardiography of the rats before sacrifice; (D) LVEF, as detected by M-mode echocardiography with parasternal long axis views ( $\mathrm{n}=10$ for the $\mathrm{SO}$ group, $\mathrm{n}=16$ for the CK group, and $\mathrm{n}=20$ for the MT group). *, $\mathrm{P}<0.05$; **, $\mathrm{P}<0.01$. I/R, ischemia/reperfusion; MI/R, myocardial ischemia/reperfusion; LVEF, left ventricular ejection fraction. 
Table 1 Parameters of echocardiogram

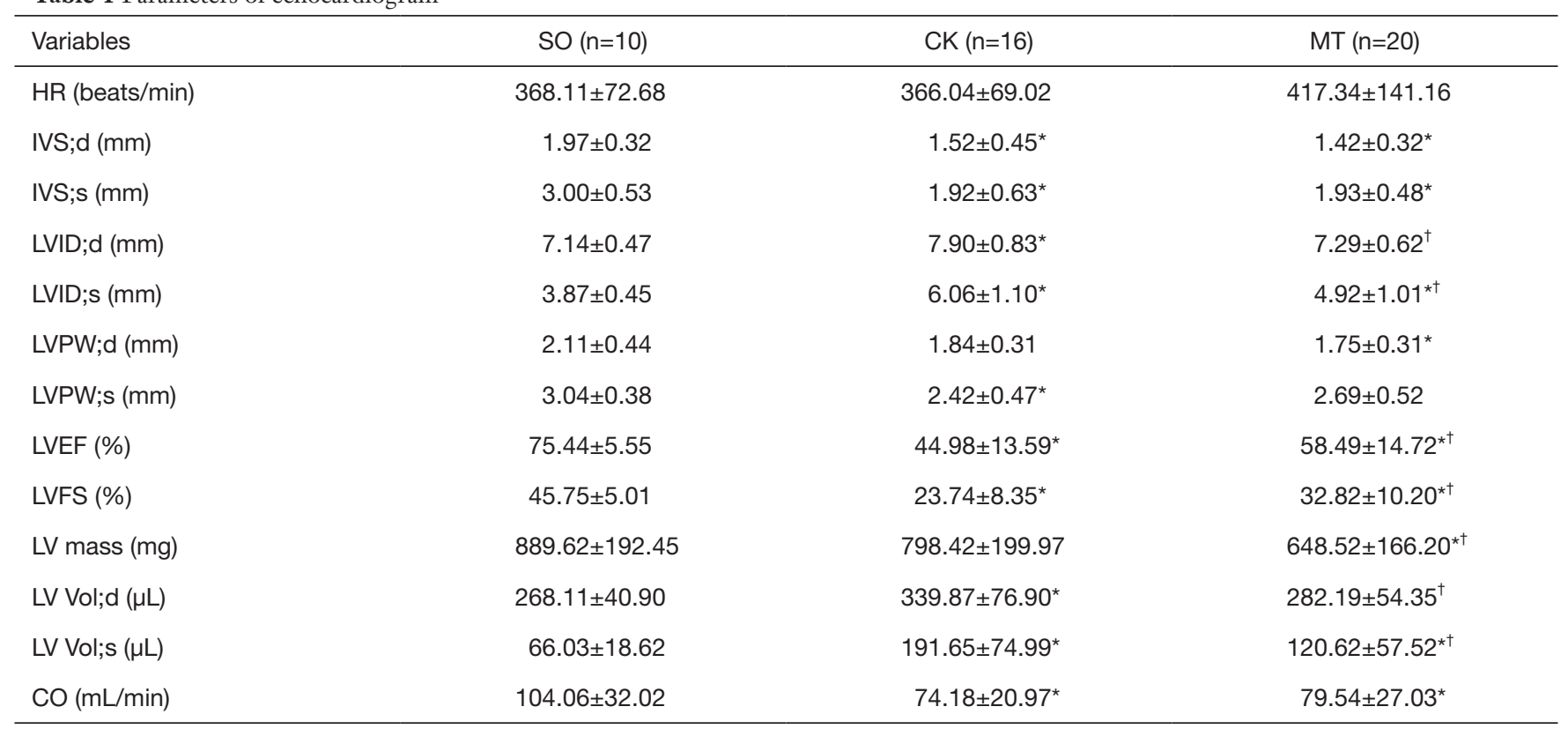

Data were expressed as the mean \pm SEM. *, $\mathrm{P}<0.05$ vs. SO; ${ }^{\dagger}, \mathrm{P}<0.05$ vs. CK. HR, heart rate; IVS; d, interventricular septum (diastolic); IVS;s, interventricular septum (systolic); LVID;d, end-diastolic left ventricular internal diameter; LVID;s, end-systolic left ventricular internal diameter; LVPW;d, left ventricular posterior wall (diastolic); LVPW;s, left ventricular posterior wall (systolic); LVEF, left ventricular ejection fraction; LVFS, left ventricular fractional shortening; LV mass, left ventricular mass; LV Vol;d, end-diastolic left ventricular volume; LV Vol;s, end-systolic left ventricular volume; $\mathrm{CO}$, cardiac output.

of PDGFRs and the phosphorylated form of CFs were measured after co-culture with M2b cells or culture with their supernatant (Figure 4C). These results showed that M2b macrophages significantly inhibited PDGFR $\alpha$ and PDGFR $\beta$ phosphorylation compared to that of the blank control. A significant increase in the expression of PDGFR $\alpha$ was observed in the M2b macrophage supernatant group compared to that of the blank control. However, there were no significant differences in PDGFR $\alpha$ and PDGFR $\beta$ expression among other groups.

\section{Discussion}

MI with or without reperfusion is a major cause of chronic $\mathrm{LV}$ remodeling that eventually results in interstitial fibrosis, dilatation, impaired contraction, and life-threatening arrhythmia. The inflammatory response to tissue injury significantly influences post-I/R remodeling (15). Numerous studies have established that macrophage infiltration is especially important in cardiac repair and fibrosis (16). Cardiac macrophages, including resident tissue macrophages and monocytes recruited from the bone marrow, can be divided into the classically activated (M1) and alternatively activated (M2) types (17). In the early phase of MI/RI, a large number of monocytes infiltrate and differentiate into M1 macrophages, with augmented expression of proteinases and proinflammatory mediators. The M2 subpopulations become predominant at the later stage, with the expression of anti-inflammatory, pro-fibrotic, and angiogenic factors, and they are generally considered profibrotic cells (18).

However, M2b macrophages are an obvious exception and are different from M2a and M2c macrophages. The reason for this is that $\mathrm{M} 2 \mathrm{~b}$ macrophages modulate inflammatory immune responses but do not appear to participate actively in wound healing, and they do not cause fibrosis (19). These cells have been referred to as the regulatory macrophage $(9,19)$. Based on the phenotypic molecules expressed and the cytokines secreted by these cells, which we explored in a previous study (12), M2b macrophages maintain a balance between anti- and proinflammatory functions, which indicates that they are regulatory cells. M2b macrophages have well-described immunosuppressive activities in inflammatory diseases $(19,20)$, most of which involve IL-10 production. Several preclinical and early-stage clinical trials showed that $\mathrm{M} 2 \mathrm{~b}$ 
A

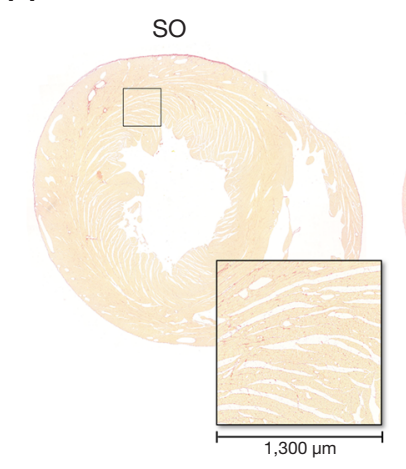

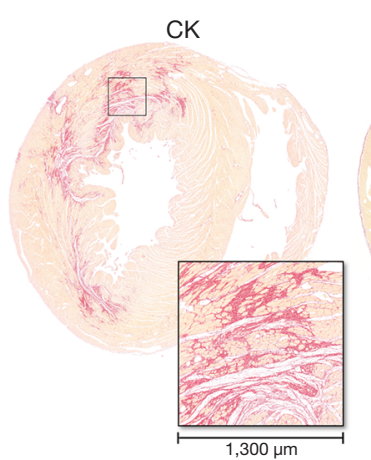

C
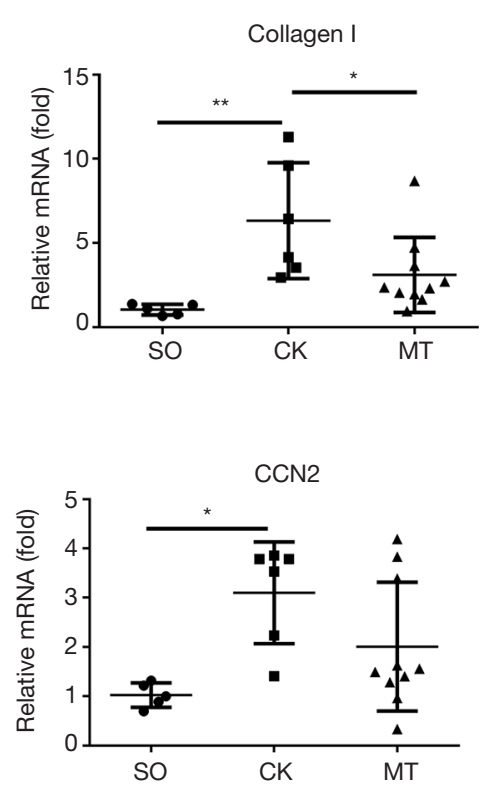

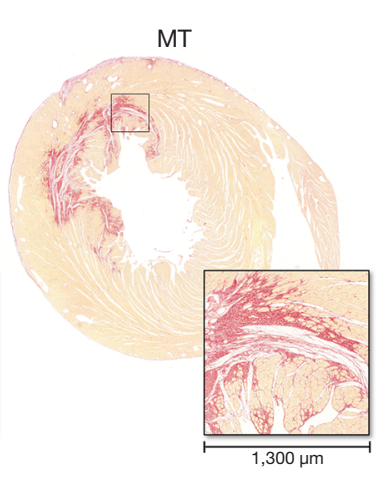

B
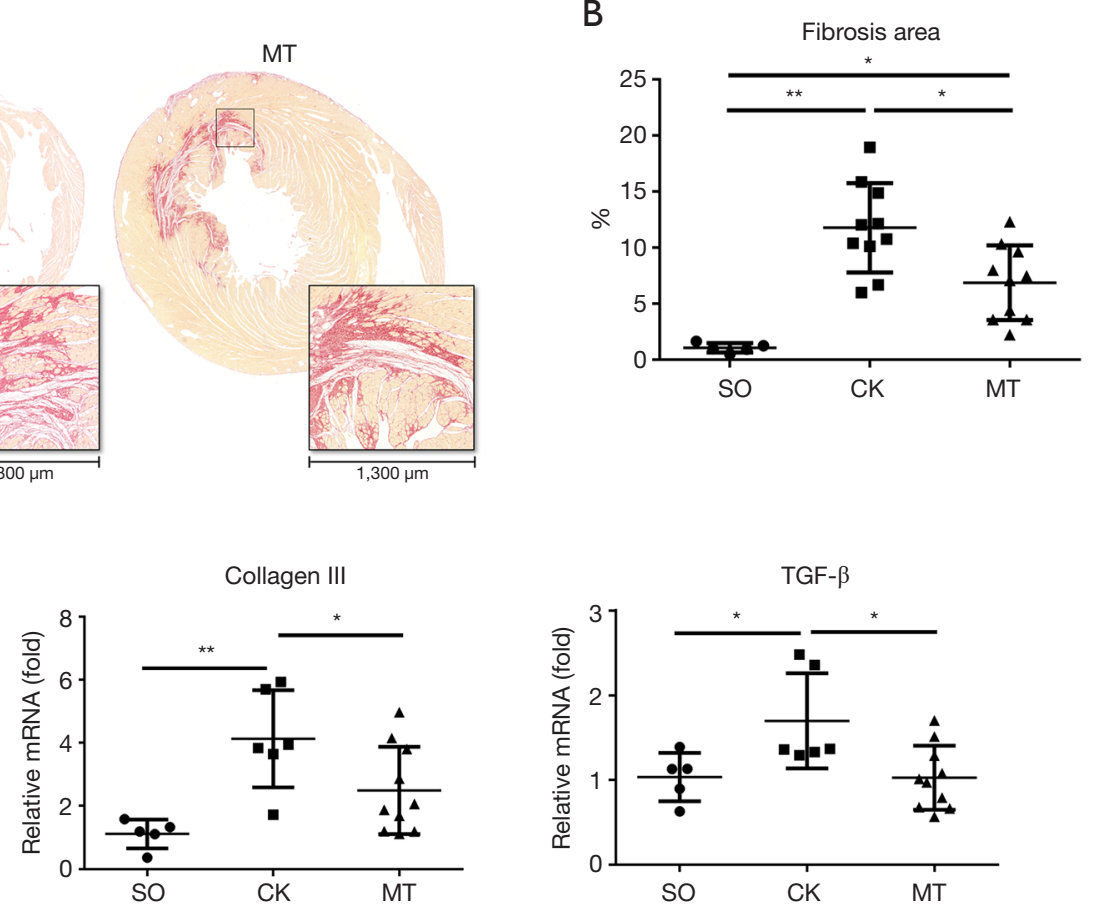

Figure 2 Transplantation of M2b macrophages alleviated myocardial fibrosis after I/R injury. (A) Histopathological features of collagen deposition based on the Sirius red staining of heart sections from a rat model (18x and 160x); (B) quantification of the Sirius red staining ( $\mathrm{n}=5$ for the SO group and n=10 for the CK group and the MT group); (C) myocardial mRNA expression of collagen I, collagen III, TGF- $\beta$, CCN2 and HGF in the different groups ( $\mathrm{n}=5$ for the SO group, $\mathrm{n}=6$ for the CK group, and $\mathrm{n}=10$ for the MT group). *, $\mathrm{P}<0.05$; ${ }^{* *}, \mathrm{P}<0.01$.

macrophage therapy has significant therapeutic potential in renal transplantation $(21,22)$. However, experimental studies investigating the role of $\mathrm{M} 2 \mathrm{~b}$ subpopulations in heart injury have not been performed. In our previous study, we demonstrated that $\mathrm{M} 2 \mathrm{~b}$ macrophages can ameliorate tissue injury in the setting of early MI/RI. We now propose that these M2b macrophages possess a second novel activity that allows them to heal I/R injury and reduce heart remodeling.

In this study, we found that the transplantation of $\mathrm{M} 2 \mathrm{~b}$ macrophages into rats subjected to MI/RI injury increased their survival, improved cardiac function, reduced cardiac fibrosis and alleviated myocardial remodeling, relative to the CK group. During the surgical procedure of model establishment, we excluded rats with severe arrhythmia, cardiac arrest, or respiratory failure. There were four, four and two rats that showed these effects in the CK, MT and SO groups, respectively. After excluding these rats with acute serious events, different survival rates were apparent among the groups. The rats receiving $\mathrm{M} 2 \mathrm{~b}$ macrophages (MT group) maintained a $100 \%$ survival rate. However, the survival rate of rats receiving normal saline (CK group) was $80 \%(16 / 20)$. Heart failure was the most likely cause of death in the CK group, manifesting as severe hypoactivity, shortness of breath, and weight 
A

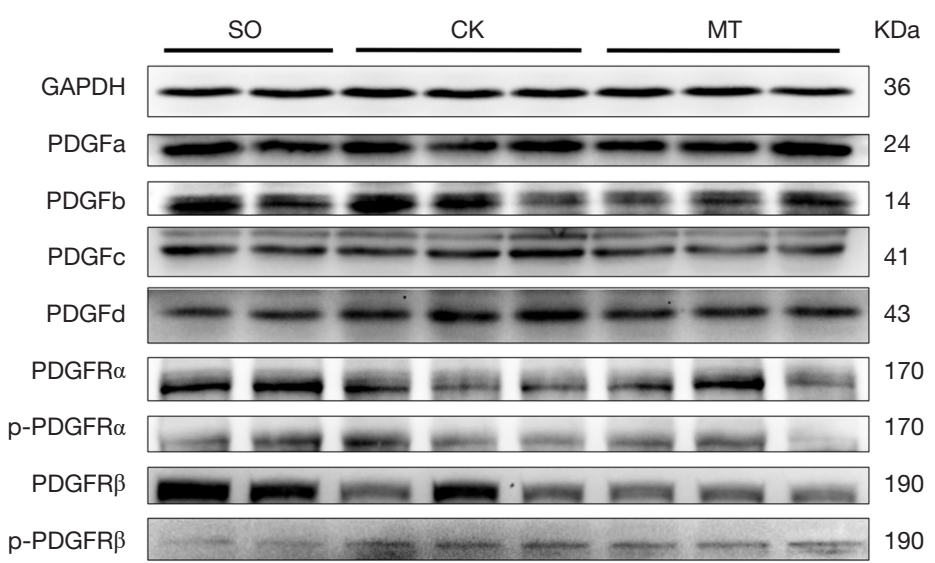

B
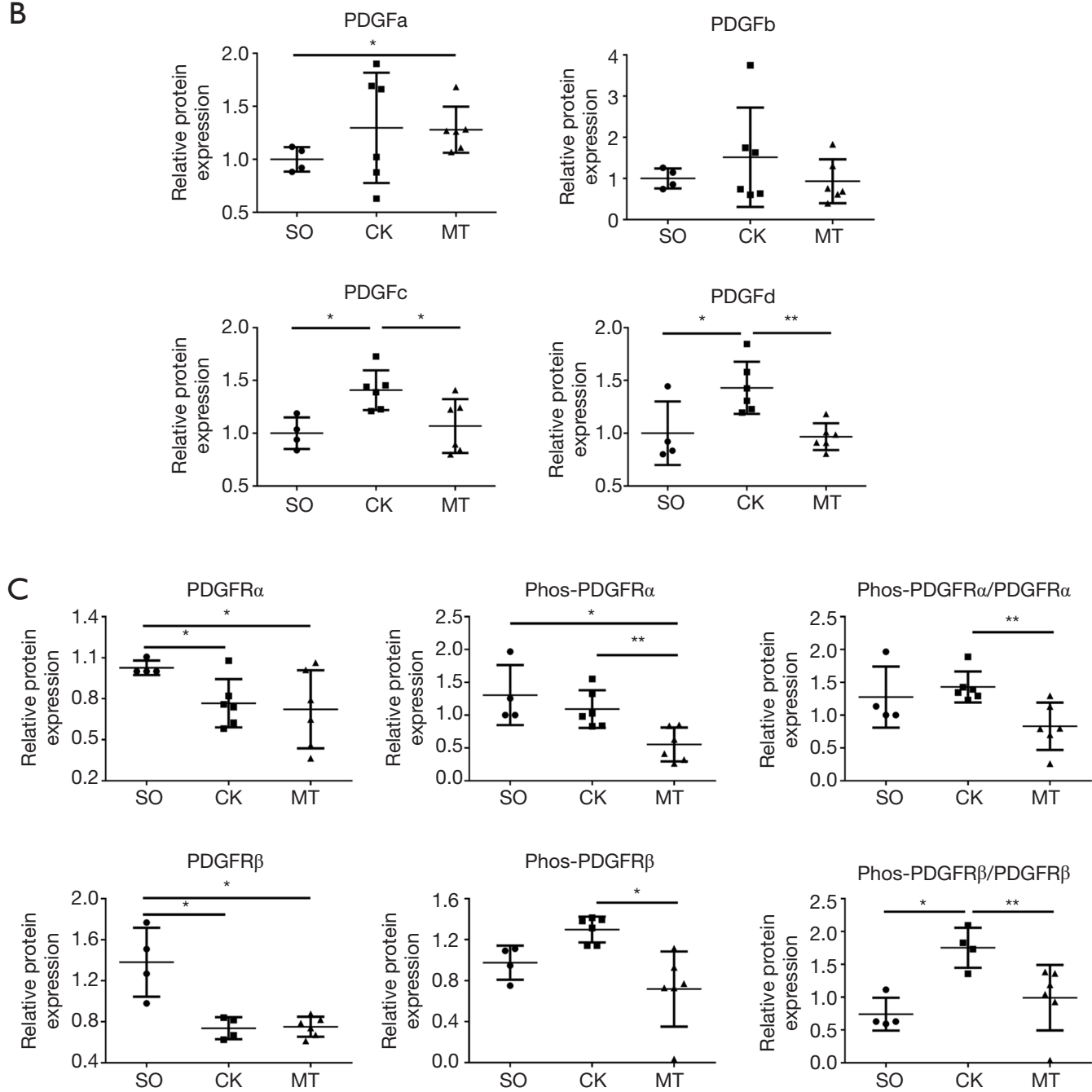

Figure $3 \mathrm{M} 2 \mathrm{~b}$ macrophages inhibited the expression of PDGFs and the kinase activation of PDGFRs in heart tissue. (A) Western blots of PDGFs, PDGFRs and Phos-PDGFRs (p-PDGFR $\alpha$ at Tyr720; p-PDGFR $\beta$ at Tyr740) in rat hearts; (B) expression of PDGFs in rat hearts; (C) expression and phosphorylation of PDGFRs in rat hearts. $n=4$ for the SO group, $n=6$ for the CK group, and $n=6$ for the MT group. *, $\mathrm{P}<0.05 ;{ }^{* *}, \mathrm{P}<0.01$. PDGF, platelet-derived growth factor; PDGFR, platelet-derived growth factor receptor. 
A $\alpha-S M A$ DAPI

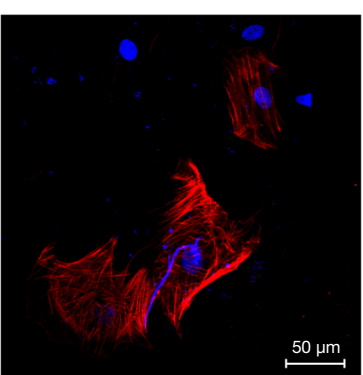

NT

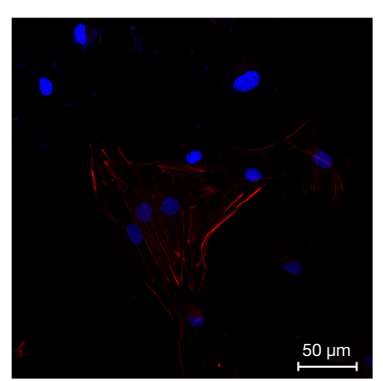

M2b co-culture

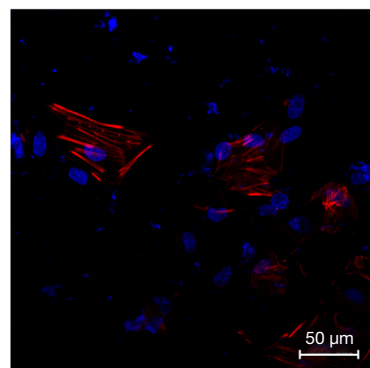

M2b supernatant
B

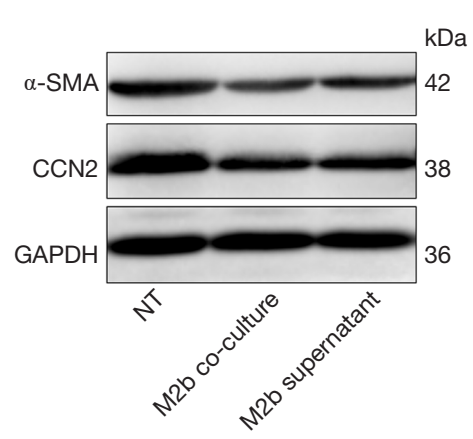

C
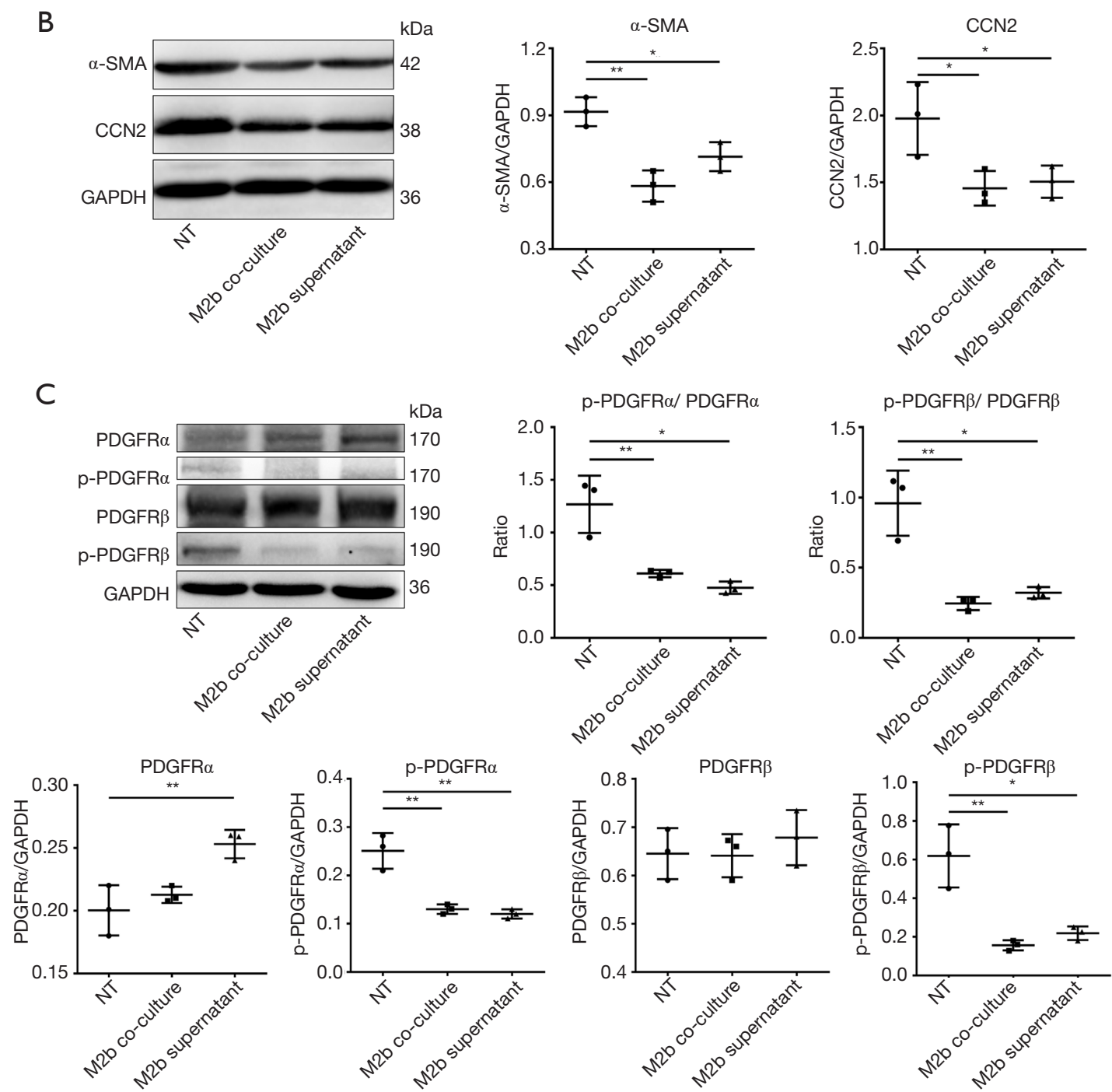

Figure $4 \mathrm{M} 2 \mathrm{~b}$ macrophages inhibited the kinase activation of PDGFRs of CFs. CFs were co-cultured with M2b macrophages or cultured with M2b macrophage supernatant or the same volume of culture medium (blank control, NT). (A) Expression of $\alpha$-SMA evaluated with immunofluorescence staining (400x); (B) expression of $\alpha$-SMA and CCN2 with western blot analysis; (C) expression and phosphorylation of PDGFRs in CFs with western blot analysis. The data in (B) and (C) are representative of three independent experiments. *, $\mathrm{P}<0.05$; ${ }^{* *}$, $\mathrm{P}<0.01$. PDGFR, platelet-derived growth factor receptor; $\mathrm{CF}$, cardiac fibroblast. 
loss. Although decreased cardiac function and substantial fibrosis could also be observed in the MT group, the degree was significantly reduced compared with the CK group. The transplantation of $\mathrm{M} 2 \mathrm{~b}$ macrophages may prevent extremely severe heart failure by protecting cardiac function and reducing ventricular remodeling, thus preventing death in the 2-week time frame of this experiment. In addition, M2b macrophages could reduce cardiomyocyte necrosis and apoptosis early after an I/R injury (12). More strikingly, M2b macrophages protected against remodeling by regulating CF activation, which is clear from the results, particularly from the in vitro experiments. These results showed that compared to the control, the expression of $\alpha$-SMA and CCN2 were significantly depressed by coculturing with $\mathrm{M} 2 \mathrm{~b}$ macrophages or culturing with $\mathrm{M} 2 \mathrm{~b}$ macrophages supernatant, which are typical markers of CF activation (23). Moreover, our previous in vitro studies have demonstrated that inhibition of PDGFRs could significantly reduce the expression of $\alpha$-SMA in CFs (14).

CFs are widely accepted to be responsible for cardiac fibrosis (24). After injury, cardiomyocytes are lost to cell death and CFs are activated to initiate reparative fibrosis (13). However, a hyperactive repair program has been hypothesized to cause pathological fibrosis. In this study, BMDMs were induced to differentiate into M2b macrophage subtypes in vitro and then transplanted into a rat model of MI/RI. Although we observed that transplantation of $\mathrm{M} 2 \mathrm{~b}$ macrophages improved cardiac function and reduced cardiac fibrosis, whether and how the M2b macrophages were kept activated after transplantation remain to be investigated. One possible explanation is that the CFs awaken quite soon after the cardiac injury, then intimately participate in all critical phases, consisting of inflammation, proliferation of non-myocytes and scar maturation (13). Following the transplantation of $\mathrm{M} 2 \mathrm{~b}$ macrophages into the injured myocardium, the expression of various cytokines and pro-fibrotic factors is downregulated in $\mathrm{CF}$, leading to decreased proliferation of these cells and ultimately, the remission of fibrosis.

Activated PDGFRs are the key driving forces in CFs activation and differentiation to generate MFs $(1,13)$, which secrete elevated levels of collagens and other extracellular matrix (ECM) proteins (25). Studies have shown that following MI, the enhanced expression of the PDGFRs occurs concomitantly with inflammatory and fibrogenic responses (26) and that blocking the PDGFRs can reduce the fibrosis of both infarcted and non-infarcted myocardium $(27,28)$. We found that in vivo, M2b macrophages suppressed the fibrosis of both the ischemic and the nonischemic myocardium. Furthermore, phosphorylation of PDGFR $\alpha$ and PDGFR $\beta$, rather than their expression level, were significantly decreased by M2b macrophage transplantation. Our in vitro study further indicates that the activation of PDGFRs in CFs was decreased by M2b macrophages.

The ligand-dependent derepression of the PDGFR kinase activity is a major mode by which PDGFRs are activated (29). Briefly, the interaction of dimeric PDGF isoforms with PDGFRs results in the dimerization and autophosphorylation of the receptors, which is followed by the activation of the PDGFR signaling system (30). We can see from the in vivo results that the M2b macrophage inhibited the expression of PDGFc and PDGFd, the which could respectively bind to PDGFR $\alpha$ and PDGFR $\beta$ in the dimer form (31). The result is consistent with that of the PDGFRs. The expression of PDGFs in CFs was not tested because that $\mathrm{CF}$ is the primary source of receptors rather than ligands of PDGFR/PDGF system. However, further studies are needed to fully elucidate the relations between M2b macrophages, PDGFR signaling, and CFs due to the complexities of macrophage subtypes and the interrelations between different signaling pathways.

There are some limitations of this study that should be taken into consideration. First, the mechanism underlying the relationship between M2b macrophages and PDGFRs is unclear. Existing PDGFR inhibitors are mostly synthetic $(32,33)$, such as imatinib (14), sorafenib (34), and pazopanib (35). Others inhibitors are anti-PDGFR antibody neutralizers, aptamers and siRNAs (31). However, none of these molecules is supposed to be produced by $\mathrm{M} 2 \mathrm{~b}$ macrophages, as far as we know. In some preclinical studies, some microRNAs (miRNAs) were found to be involved in the inhibition of the PDGFR/ PDGF system, which could be produced as the contents of extracellular vesicles. However, there is no direct evidence for this hypothesis, and this is the largest limitation of this study. Second, no other macrophage subsets were used as a control to demonstrate the specificity of the beneficial effects of M2b macrophages. Part of the reason for this approach is that we extensively studied this topic in our previous research, from the acute phase after MI/ $\mathrm{R}$ to convalescence. Finally, although the activation of fibroblasts has been shown to be inhibited by M2b macrophages and is considered to be the main reason for reducing fibrosis, more explorations need to be completed to exclude the confounding factor-the reduction of acute 


\section{Page 10 of 11}

heart injury by $\mathrm{M} 2 \mathrm{~b}$ macrophages.

In conclusion, this study showed that M2b macrophages protect against myocardial remodeling after I/R injury. The direct effects of M2b macrophages on the CFs may be responsible for the reduced fibrosis. Depressed kinase activation of PDGFR may be part of the mechanism through limiting CFs activation. Taken together, the results of this study suggest that therapy with $\mathrm{M} 2 \mathrm{~b}$ macrophages may be a potential treatment strategy for heart damage and remolding caused by I/R injury.

\section{Acknowledgments}

Funding: This work was supported by the National Key R\&D Program of China (2017YFC1105000), the National Natural Science Foundation of China (81770319, 81900294), the PhD Start-up Fund of Natural Science Foundation of Guangdong Province (No. 2018A030310403 for WLX).

\section{Footnote}

Reporting Checklist: The authors have completed the ARRIVE reporting checklist. Available at http://dx.doi. org/10.21037/atm-20-2788

Data Sharing Statement: Available at http://dx.doi. org/10.21037/atm-20-2788

Peer Review File: Available at http://dx.doi.org/10.21037/ atm-20-2788

Conflicts of Interest: All authors have completed the ICMJE uniform disclosure form (available at http://dx.doi. org/10.21037/atm-20-2788). The authors have no conflicts of interest to declare.

Ethical Statement: The authors are accountable for all aspects of the work in ensuring that questions related to the accuracy or integrity of any part of the work are appropriately investigated and resolved. Experiments were performed under a project license (No. gdpulacSPF2017026) granted by the Animal Ethical Committee of Guangdong Pharmaceutical University, in compliance with the relevant guidelines for the care and use of laboratory animals. This article does not contain any studies with humans performed by any of the authors.
Yue et al. M2b macrophages protect against myocardial remodeling

Open Access Statement: This is an Open Access article distributed in accordance with the Creative Commons Attribution-NonCommercial-NoDerivs 4.0 International License (CC BY-NC-ND 4.0), which permits the noncommercial replication and distribution of the article with the strict proviso that no changes or edits are made and the original work is properly cited (including links to both the formal publication through the relevant DOI and the license). See: https://creativecommons.org/licenses/by-nc-nd/4.0/.

\section{References}

1. Leask A. Getting to the heart of the matter: new insights into cardiac fibrosis. Circ Res 2015;116:1269-76.

2. Prabhu SD, Frangogiannis NG. The biological basis for cardiac repair after myocardial infarction: from inflammation to fibrosis. Circ Res 2016;119:91-112.

3. Kania G, Blyszczuk P, Eriksson U. Mechanisms of cardiac fibrosis in inflammatory heart disease. Trends Cardiovasc Med 2009;19:247-52.

4. Wang L, Li YL, Zhang CC, et al. Inhibition of Tolllike receptor 2 reduces cardiac fibrosis by attenuating macrophage-mediated inflammation. Cardiovasc Res 2014;101:383-92.

5. Falkenham A, de Antueno R, Rosin N, et al. Nonclassical resident macrophages are important determinants in the development of myocardial fibrosis. Am J Pathol 2015;185:927-42.

6. Fujiu K, Wang J, Nagai R. Cardioprotective function of cardiac macrophages. Cardiovasc Res 2014;102:232-9.

7. Mantovani A, Sozzani S, Locati M, et al. Macrophage polarization: tumor-associated macrophages as a paradigm for polarized M2 mononuclear phagocytes. Trends Immunol 2002;23:549-55.

8. Nahrendorf M, Swirski FK. Monocyte and macrophage heterogeneity in the heart. Circ Res 2013;112:1624-33.

9. Mosser DM, Edwards JP. Exploring the full spectrum of macrophage activation. Nat Rev Immunol 2008;8:958-69.

10. Steffens S, Montecucco F, Mach F. The inflammatory response as a target to reduce myocardial ischaemia and reperfusion injury. Thromb Haemost 2009;102:240-7.

11. Ma Y, Halade GV, Zhang J, et al. Matrix metalloproteinase-28 deletion exacerbates cardiac dysfunction and rupture after myocardial infarction in mice by inhibiting M2 macrophage activation. Circ Res 2013;112:675-88.

12. Yue $\mathrm{Y}$, Yang $\mathrm{X}$, Feng $\mathrm{K}$, et al. M2b macrophages reduce early reperfusion injury after myocardial ischemia in mice: 
a predominant role of inhibiting apoptosis via A20. Int J Cardiol 2017;245:228-35.

13. Travers JG, Kamal FA, Robbins J, et al. Cardiac fibrosis: the fibroblast awakens. Circ Res 2016;118:1021-40.

14. Wang LX, Yang X, Yue Y, et al. Imatinib attenuates cardiac fibrosis by inhibiting platelet-derived growth factor receptors activation in isoproterenol induced model. PLoS One 2017;12:e0178619.

15. Frangogiannis NG. Regulation of the inflammatory response in cardiac repair. Circ Res 2012;110:159-73.

16. Nahrendorf M, Pittet MJ, Swirski FK. Monocytes: protagonists of infarct inflammation and repair after myocardial infarction. Circulation 2010;121:2437-45.

17. Martinez FO, Sica A, Mantovani A, et al. Macrophage activation and polarization. Front Biosci 2008;13:453-61.

18. Wynn TA. Cellular and molecular mechanisms of fibrosis. J Pathol 2008;214:199-210.

19. Fleming BD, Mosser DM. Regulatory macrophages: setting the threshold for therapy. Eur J Immunol 2011;41:2498-502.

20. Gerber JS, Mosser DM. Reversing lipopolysaccharide toxicity by ligating the macrophage Fc gamma receptors. J Immunol 2001;166:6861-8.

21. Broichhausen C, Riquelme P, Geissler EK, et al. Regulatory macrophages as therapeutic targets and therapeutic agents in solid organ transplantation. Curr Opin Organ Transplant 2012;17:332-42.

22. Hutchinson JA, Riquelme P, Sawitzki B, et al. Cutting Edge: Immunological consequences and trafficking of human regulatory macrophages administered to renal transplant recipients. J Immunol 2011;187:2072-8.

23. Porter KE, Turner NA. Cardiac fibroblasts: at the heart of myocardial remodeling. Pharmacol Ther 2009; 123:255-78.

24. Ma Y, de Castro Bras LE, Toba H, et al. Myofibroblasts and the extracellular matrix network in postmyocardial infarction cardiac remodeling. Pflugers Arch

Cite this article as: Yue Y, Huang S, Li H, Li W, Hou J, Luo L, Liu Q, Wang C, Yang S, Lv L, Shao J, Wu Z. M2b macrophages protect against myocardial remodeling after ischemia/reperfusion injury by regulating kinase activation of platelet-derived growth factor receptor of cardiac fibroblast. Ann Transl Med 2020;8(21):1409. doi: 10.21037/atm-20-2788
2014;466:1113-27.

25. Kawaguchi M, Takahashi M, Hata T, et al. Inflammasome activation of cardiac fibroblasts is essential for myocardial ischemia/reperfusion injury. Circulation 2011;123:594-604.

26. Zhao W, Zhao T, Huang V, et al. Platelet-derived growth factor involvement in myocardial remodeling following infarction. J Mol Cell Cardiol 2011;51:830-8.

27. Liu C, Zhao WY, Meng WX, et al. Platelet-derived growth factor blockade on cardiac remodeling following infarction. Mol Cell Biochem 2014;397:295-304.

28. Zhao T, Zhao W, Chen Y, et al. Platelet-derived growth factor-D promotes fibrogenesis of cardiac fibroblasts. Am J Physiol Heart Circ Physiol 2013;304:H1719-26.

29. Kazlauskas A. PDGFs and their receptors. Gene 2017;614:1-7.

30. Andrae J, Gallini R, Betsholtz C. Role of platelet-derived growth factors in physiology and medicine. Genes Dev 2008;22:1276-312.

31. Appiah-Kubi K, Wang Y, Qian H, et al. Platelet-derived growth factor receptor/platelet-derived growth factor (PDGFR/PDGF) system is a prognostic and treatment response biomarker with multifarious therapeutic targets in cancers. Tumour Biol 2016;37:10053-66.

32. Galluzzi L, Vacchelli E, Fridman WH, et al. Trial Watch: Monoclonal antibodies in cancer therapy. Oncoimmunology 2012;1:28-37.

33. Schwickart M, Mehrzai F, Pearson J, et al. Identification and elimination of target-related matrix interference in a neutralizing anti-drug antibody assay. J Immunol Methods 2014;403:52-61.

34. Norton KA, Han Z, Popel AS, et al. Antiangiogenic cancer drug sunitinib exhibits unexpected proangiogenic effects on endothelial cells. Onco Targets Ther 2014;7:1571-82.

35. Lamba G, Ambrale S, Lee B, et al. Recent advances and novel agents for gastrointestinal stromal tumor (GIST). J Hematol Oncol 2012;5:21. 